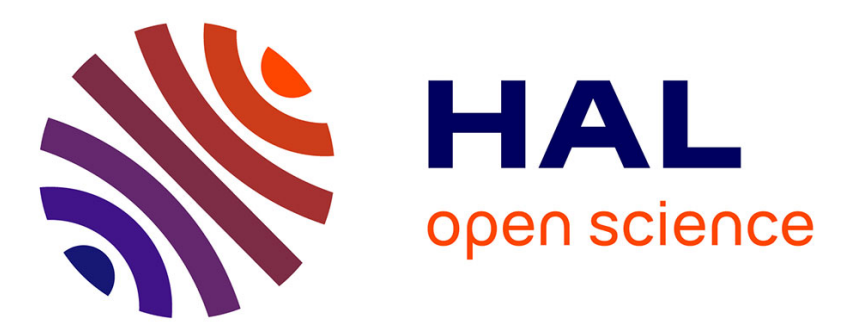

\title{
Determination of a tactile feedback strategy for use in robotized percutaneous procedures
}

Rui Zhu, Lennart Rubbert, Pierre Renaud, Ulrich Mescheder

\section{To cite this version:}

Rui Zhu, Lennart Rubbert, Pierre Renaud, Ulrich Mescheder. Determination of a tactile feedback strategy for use in robotized percutaneous procedures. 41st Annual International Conference of the IEEE Engineering in Medicine and Biology Society (EMBC), Jul 2019, Berlin, Germany. 10.1109/EMBC.2019.8857235 . hal-03254580

\section{HAL Id: hal-03254580 \\ https://hal.science/hal-03254580}

Submitted on 8 Jun 2021

HAL is a multi-disciplinary open access archive for the deposit and dissemination of scientific research documents, whether they are published or not. The documents may come from teaching and research institutions in France or abroad, or from public or private research centers.
L'archive ouverte pluridisciplinaire HAL, est destinée au dépôt et à la diffusion de documents scientifiques de niveau recherche, publiés ou non, émanant des établissements d'enseignement et de recherche français ou étrangers, des laboratoires publics ou privés. 


\title{
Determination of a tactile feedback strategy for use in robotized percutaneous procedures
}

\author{
Rui Zhu ${ }^{1}$, Lennart Rubbert ${ }^{2}$, Pierre Renaud ${ }^{2}$ and Ulrich Mescheder ${ }^{1}$
}

\begin{abstract}
Remote manipulation in robotized percutaneous procedures can offer increased safety to radiologists as well as patients. Providing feedback to the radiologist on needle-tissue interactions is however mandatory in addition to the medical images. A tactile feedback strategy is developed in this paper. Two types of information are considered: tissue puncture and nature of tissues. A haptic device is developed for that purpose, using a tactile display to provide information. Adequate signals are identified experimentally, with analysis of reaction times and the ability to discriminate one information from the other.
\end{abstract}

\section{INTRODUCTION}

Interventional radiology and more generally image-guided surgery is being developed nowadays [1], [2]. Real-time imaging is a great advantage to make a diagnosis and also to perform surgery, beyond what is visible directly or with cameras. Percutaneous procedures, performed by using needles, are one of the most developed techniques. It allows biopsy and also minimally-invasive treatment of tumors using for instance radiofrequency or cryoablation [3]. The development of percutaneous procedures is however hampered by two main issues. First, the use of X-Ray based imaging devices represents a risk for the radiologist in case of manual manipulation. As a solution, commercial devices and research prototypes are developed to offer a safe remote manipulation [4], [5]. Second, MRI scanner geometry, with a long and narrow bore, makes procedures very difficult in terms of accessibility and accuracy, whereas such imaging modality is well adapted for soft tissue imaging [6]. Robotized remote manipulation is again a solution to improve task feasibility [7]. As a consequence, some solutions are now being developed to offer at the same time assistance in X-Ray and MR-based imaging devices [8], [9]. This paper is dedicated to the development of such solutions, following the workflow analysis and preliminary work presented in [5]. During a percutaneous procedure, the radiologist cannot be sure that the whole needle is visible in 2D images. For safety reasons, interaction forces between the needle and the tissues are therefore being used by the radiologist today to estimate the tip position. It is possible to have a direct measurement of interaction forces at the needle tip [10], in particular to detect punctures as encountered in these non-vascular interventions. In this paper we investigate how to bring information on the needle-tissue interactions in such a case. More specifically, we assess different strategies to provide

*This work was supported by the INTERREG Upper Rhine program from the ERDF (European Regional Development Fund), SPIRITS project.

${ }^{1}$ Hochschule Furtwangen rui.zhu@hs-furtwangen.de

2 AVR-ICube, Strasbourg University, INSA Strasbourg lennart.rubberteinsa-strasbourg.fr

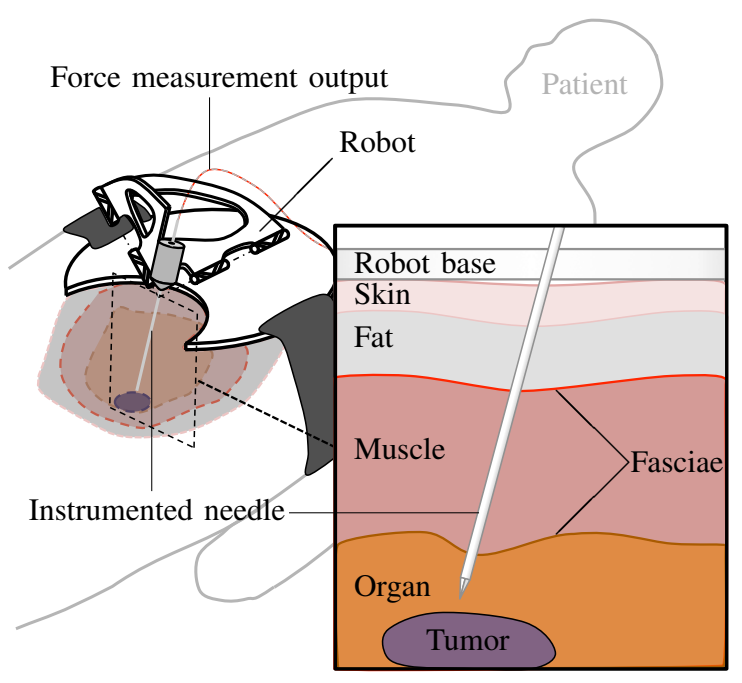

Fig. 1. Schematic representation of robotized percutaneous procedure, with different tissues encountered during needle insertion.

the radiologist with information on needle-tissue interaction using tactile feedback. Our proposition is to bring to the user two types of information. The first one is the occurence of tissue puncture, and the second one is the nature of tissues. A haptic device is developed for that purpose, using a tactile display to provide information. Adequate signals are identified experimentally, with analysis of reaction times and the ability to discriminate one information related to puncture, and another one to the nature of tissues.

The paper falls into four parts. First, the needle insertion task is being analyzed in section II to justify the feedback provided to the radiologist. Literature review is then used to select a tactile feedback strategy. In section III, the experimental device built for evaluation is presented with the associated protocol. Results are then introduced and discussed in section IV. Finally, perspectives are introduced in section $\mathrm{V}$.

\section{ELABORATION OF FEEDBACK SCENARIO}

\section{A. Task analysis}

Procedures on abdomen and thorax represent a significant fraction of percutaneous tasks, because of the related occurence of cancers [11]. Task analysis is thus introduced in that context. Patient-mounted robotic devices offer the possibility to partially compensate for patient motions, and also limit accessibility issues related to MRI scanners [12]. As shown in Fig. 1, needle manipulation is thus considered to be performed remotely, using a teleoperation scheme, with the slave robot positioned on top of the patient. The 
critical phase of needle placement is the teleoperation of needle insertion by the radiologist. Mechatronic solutions exist to safely translate the needle in the tissues, for instance with compact pneumatic actuators [13]. The radiologist that manipulates a master interface needs to understand where the needle is located, and if the needle motion is appropriate. The master interface is operated close to the imaging device with a user in sterile conditions, wearing gloves.

We performed observations of clinical procedures with manual approaches in interventional radiology centers in Strasbourg, France and Mannheim, Germany. Interviews were also performed. It was concluded that two types of information are being used to complement the visual information during the insertion [5]. The first one is information about the nature of tissues. For the tasks under consideration, the needle goes through skin, fat, muscles and organs such as liver, kidney or lung, before reaching the target to be punctured, e.g. a tumor (Fig. 1). Each of these anatomical structures is characterized by stiffnesses which are significantly different [14]. Fasciae are also located between fat, muscle and organ. Their puncture is an event that is useful for the radiologist to validate that the needle has penetrated a new anatomical structure [10]. Because of image acquisition time, needle insertion is performed slowly. Typically, the needles's insertion speed does not exceed $1 \mathrm{~mm} / \mathrm{s}$. The expected accuracy of procedure is about $1 \mathrm{~mm}$. It means that the radiologist needs to react when a puncture occurred, or whenever the nature of tissue is changing, within a delay of about $1 \mathrm{~s}$.

Thanks to optical sensing techniques, it is possible to have a surgical needle with measurement of displacement and force at the tip [10]. Puncture information can then be determined reliably. In addition, we consider that an estimation of stiffness and accordingly the nature of tissue can be computed. Our goal in this paper is to determine a way to reflect to the user such information as well as the puncture information.

\section{B. State of the art}

During insertion, the radiologist usually uses the sense of sight to observe the medical image, but not in a continuous way as he or she is also observes the patient. Using sound or haptic feedback is then of interest [15], [16]. Sound feedback is often used by many other devices in the radiology room. In [10] it was proved that haptic feedback is efficient to provide the user with the puncturing information, at least using a basic voice coil system. Tactile feedback is a simple approach to provide haptic information in teleoperation as it does not require complex control of master interface. It was previously shown to be an efficient way to complement visual information in surgery [17] and also for needle insertion [18]. Different technologies can be used to create a tactile feedback [18], [19], [20], some of them being potentially compatible with the MR environment such as piezoelectric and pneumatic devices [7], [21].

Tactile information can be generated on a sensitive area such as the fingertip by exerting forces normal to the skin, or by skin stretching. In [18], [22] skin stretching is shown

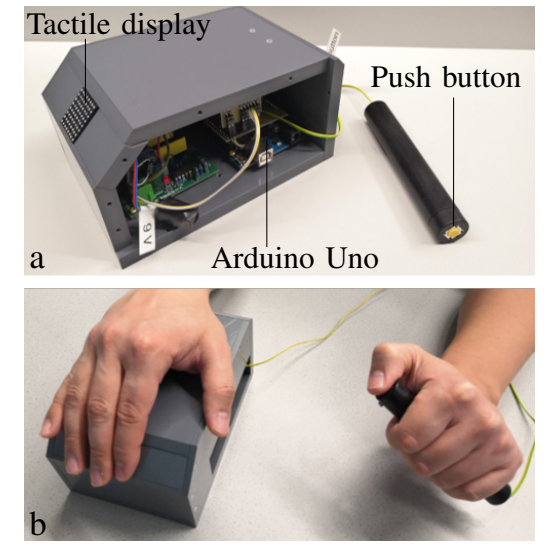

Fig. 2. Experimental set-up: (a) the interface with internal components, (b) typical configuration during evaluation.

to be of interest in medical context, with the possibility to deliver information about the direction and magnitude of a force to a user. Puncture information seems to be efficiently delivered in [18] with this technique. One potential limit is the presence of gloves, that creates an interface between the tactile actuator and the user's fingertip. In [23], the impact of gloves on the finger sensitivity was assessed in the case of static application of objects on the fingertip. No significant impact of gloves was observed on a 2-point discrimination test, when the minimum distance between two objects applied on the fingertip is determined. Our proposition is therefore to rely on the same type of force application on the skin. Qualitatively, the use of normal forces seems indeed less prone to deformation and slipping of glove material. Two types of information have to be transmitted to the user. Therefore, at least two different signals need then to be applied on the skin. Dynamic patterns have been largely developed for visually-impaired people. We investigate the use of dynamic patterns as they are easily available. Additionally, they are often based on piezoelectric actuation which is compatible with our medical environment. Considering a pattern-based tactile feedback, the question is the feasibility to have one feedback device to provide two different types of information: one for puncture, a single event, and another one to qualify the nature of tissues, which is a discrete information. No priori work related to that question exists to our knowledge. An experimental approach is thus adopted, described in the following.

\section{SET-Up AND PRotocol Elaboration}

\section{A. Experimental set-up}

The experiments are conducted using the tactile interface represented in Fig. 2. It consists of a commercial tactile actuator display (SC5, KGS Corporation) and an Arduino Uno interface, which is used to communicate with the actuator element and for data acquisition. We use a standard array of $8 \times 8$ bumps within an area of $4.41 \mathrm{~cm}^{2}$ as it allows us to compare a significant number of patterns. The dots are equally spaced by $3 \mathrm{~mm}$. They are driven by piezoelectric bimorphs, which provide a no-load stroke of $0.7 \mathrm{~mm}$. In the experiment, the subject places his/her finger on the 
display. The generated patterns are controlled by the Arduino Uno, which also receives a signal from a push button that is held by the subject in his or her other hand (Fig. 2). This allows us to assess the reaction times to changes of patterns as described below. All signals are finally recorded on a PC.

\section{B. Protocol Definition}

Due to the discussed requirements we want to reflect the presence of 4 different anatomical structures (fat, muscle, organ, tumor, excluding skin that is incised before needle penetration) and also the occurence of puncture. The two types of information have to be sent alternatively, as fascia puncture occurs between changes of tissues (Fig. 1). The test sequence is therefore built as shown in Fig. 3.

S1 designates the signal associated to a puncture event. For sake of simplicity, S1 corresponds to the dynamic activation of all bumps to create an "ON-OFF" pattern, represented by the two corresponding patterns in Fig. 4 for S1. It also maximizes the sensation under the fingertip.

As for all discussed patterns, frequency corresponds here to the inverse of the "ON-OFF"-time. The other type of information is a discrete signal, with 4 different magnitudes. The signal coding for these magnitudes can be based on only one pattern, that we then designate as a static signal, or on a sequence of patterns for a dynamic signal. Both are evaluated. Two types of static signals are considered. They are designated as S2 and S4 in Fig. 4, with cross shapes, chosen to have a very different geometry than S1. The signals S2 and S4 differ by the number of activated bumps. We decided to consider in addition four dynamic signals, chosen to be different in terms of pattern shape. S5 corresponds to a periodic activation of a cross-shaped pattern, and S3, S6 and S7 correspond to 3 basic motion patterns: a translational (S3), a rotational (S6) and an expansive motion (S7). The signals S1, S3, S5, S6 and S7 are dynamic in the sense that different parts of the tactile area are actuated over time. We consider a similar frequency for $\mathrm{S} 1$ and the other signals. We assume this does not restrict the reaction times to the change of patterns. Because of the total number of bumps, it is not possible to generate S3, S5, S6 and S7 with an equal number of frames. The frequency value therefore corresponds to the frame-rate, to make a fair comparison between signals, with equal activation times within each frame. The selected frequencies are 1, 5, 10, $15,20,30,40$ and $50 \mathrm{~Hz}$. These values are chosen to be compatible with fingertip sensitivity, and in particular with frequency changes that can be discriminated by a user using his/her fingertip [24], [25]. As a consequence, we do not assess this discrimination capacity, but consider the user ability to detect a change of nature of signal, between S1 and one of the other signals $\mathrm{Si}, \mathrm{i} \in\{2,7\}$.

One test sequence consists of switching between the signal $\mathrm{S} 1$ and one of the other signals $\mathrm{Si}, \mathrm{i} \in\{2,7\}$. We are interested in the time needed for the user to detect the signal change. We designate this as the response time, and determine it as the duration (delay) between the signal change instant and the recording of a button pressed by

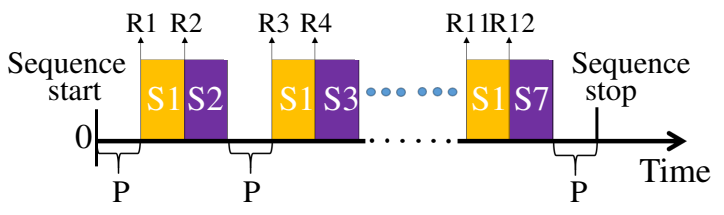

Fig. 3. Principle of the test sequence, time on the sketch is not scaled. $\mathrm{P}$ designates a pause, $\mathrm{S}$ a signal sent to the user and $\mathrm{R}$ a user response.

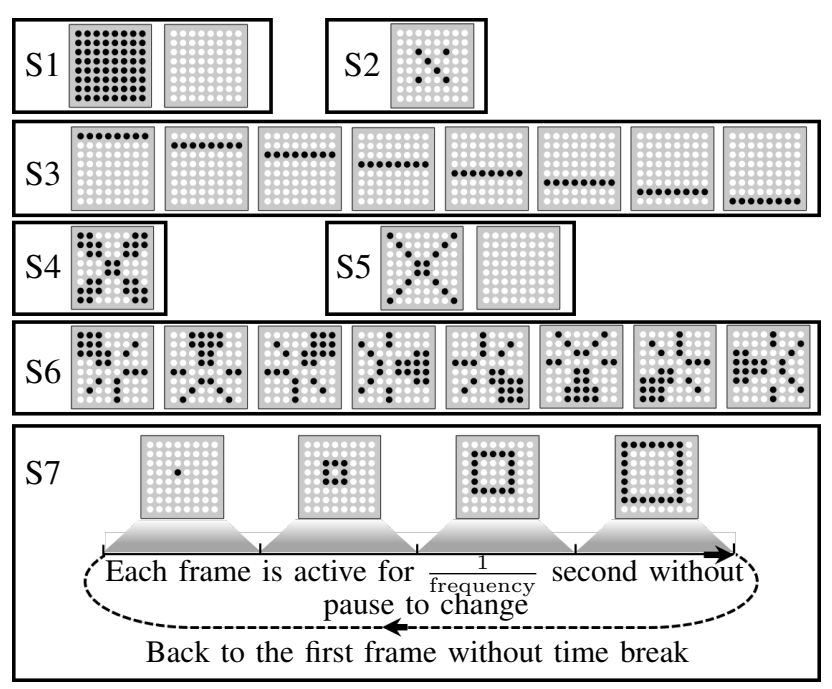

Fig. 4. Signal patterns under evaluation. Black dots designate activated bumps.

the user. We measure the response time to detect $\mathrm{S} 1$, and also from S1 to another signal. In other words, 2 types of response times are recorded for each couple of signals (S1, Si), $\mathrm{i} \in\{2,7\}$ (Fig. 3). All signals are sent for $20 \mathrm{~s}$ to make sure the user has time to react, and a 10 -second pause is introduced between each combination of 2 signals to clearly separate pairs of patterns. It was verified that the 10-second duration cannot be used by the subjects to predict the appearance of signal S1. The test sequence is finally sent to the user for the different frequencies.

During the assessment, the environment is controlled to be quiet and without any distraction. The experiment is stopped by the subject if he or she feels tired or if any finger numbness occurs. The subjects could follow the protocol without fatigue at least for 1 hour. There was no communication with the subjects during the test. The subject was only informed of the frequency used in the given sequence. The subject was free to choose the fingertip's position on the $8 \times 8$ array, even with two fingers when preferred. The same number and position of fingers is then used by the subject during all the experiments. A short learning phase is introduced, with one complete sequence of signals at $5 \mathrm{~Hz}$ that is not assessed. We expected a large masking effect from gloves, thus the impact of gloves is assessed by performing a sequence at $5 \mathrm{~Hz}$ without and with medical gloves (Prima Plus, Sänger).

The experiment results consist of the measurement of the response times. In addition, answers to a questionnaire were analyzed ot obtain a subjective rating from the test subjects. 
It is composed of 7 open questions:

1. How do you like to place your finger on the display?

2. Which pattern is the easiest to recognize?

3. Besides the recognition of the pattern, which change of signal is the easiest one for you to identify?

4. Which type of signal is easier to detect: static or dynamic? 5. Is it easier to sense a signal composed of a large number of activated bumps?

6. Is the detection of a signal change easier with a higher frequency of signals?

7. What is the frequency range you prefer for detection of signal change?

\section{RESUlTS AND Discussion}

\section{A. Results}

Assessment was run with 7 male voluntary subjects, with age ranging from 30 to 53 years. All the subjects were visually healthy and without specific experience or skills in using tactile devices before the test. They did not exercise a profession which requires a precise sensation with fingertips. Results in terms of response time to the signal S1 and response time to switching from signal $\mathrm{S} 1$ to a signal $\mathrm{Si}$, $\mathrm{i} \in\{2,7\}$ are represented in Fig. 5 and Fig. 6. For sake of visibility, Fig. 6 represents the average response time among all users without and with gloves (only tested at $5 \mathrm{~Hz}$ ). The analysis of user answers to the questionnaire leads to the following subjective statements:

1. They prefer to have their fingertips covering the whole active surface. Depending on the fingertip size they preferred to place one ( 2 subjects out of 7$)$ or two fingers $(5$ subjects out of 7) on the active surface.

2. The signal corresponding to a translation motion (S3) was the one which structure the testers could recognize most easily (6 subjects out of 7).

3. The "ON-OFF" sequence as used in S1 was considered obvious to detect (all subjects).

4. Dynamic signals were easier detected than static signal (all subjects).

5. A large number of bumps provided better detectability ( 6 subjects out of 7). For a reduced number of activated bumps, or non-uniform distributed bumps signal changes were often missed.

6. Frequencies below $25 \mathrm{~Hz}$ are preferred (all subjects).

7. The range from 10 to $25 \mathrm{~Hz}$ was for the testers the most adequate and the most preferred frequency (all subjects).

\section{B. Discussion}

The choice of $\mathrm{S} 1$ for the puncture information is supported by the subjective statements in respect to question \#3 and \#5 tending to show such a signal is considered easy to be perceived. Fig. 5 shows the quantitative response time to S1 is distributed between $0.18 \mathrm{~s}$ and $2.9 \mathrm{~s}$ with the majority (about 94\%) of recognitions faster than $1 \mathrm{~s}$. The average response time is slightly decreasing with the frequency. It is interesting to note that only $5.8 \%$ of all recordings are above $1 \mathrm{~s}$. Thus we conclude that $\mathrm{S} 1$ is acceptable at all frequencies

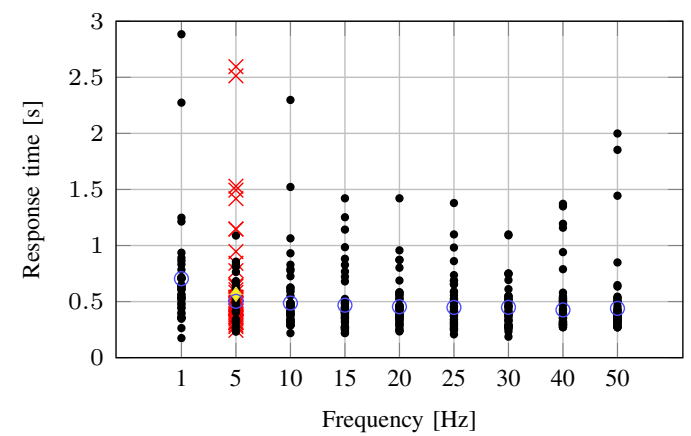

- Without gloves $\times$ With gloves $\bigcirc$ Average without gloves $\triangleright$ Average with gloves

Fig. 5. Evolution with the frequency of the response time to the activation of the signal S1 (R1, R3, ... R11 in Fig. 3) .

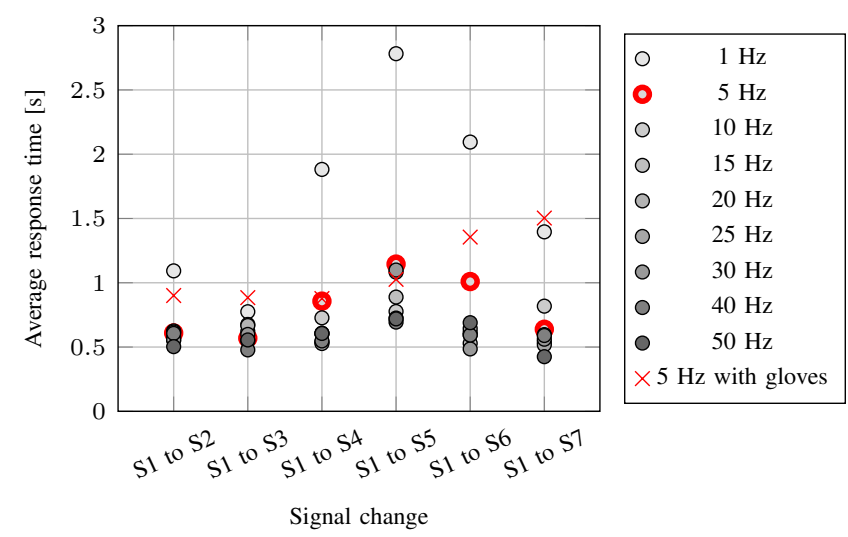

Fig. 6. Evolution of the average user response time to the switch from the signal $\mathrm{S} 1$ to the signal $\mathrm{Si}, \mathrm{i} \in\{2,7\}$ (R2, R4,..R12 in Fig. 3).

to warn the user about a puncture occurence.

Response time for the change of signals is generally decreasing with increasing frequency. Values of response time for $1 \mathrm{~Hz}$ and $5 \mathrm{~Hz}$ can be twice of those obtained at higher frequencies. Dynamic signals are preferred to static signals by subjects (comment \#4), even though it is not clearly visible in terms of response time by comparing results for $\mathrm{S} 2, \mathrm{~S} 4$ to S3, S5, S6, S7.

From the four dynamic signals, the combination S1 to S3 shows the smallest spread of the response time, and the average of response time is lower than $1 \mathrm{~s}$. This is in accordance with comments \#1 and \#2. It may be related to the robustness of the pattern to the position of the finger because of the line shape. Considering that the frequencies below $25 \mathrm{~Hz}$ are preferred (comment \#6), and that we could generate 4 signals of distinct frequencies from 10 to $25 \mathrm{~Hz}$, it seems feasible to transfer to the user the nature of tissues with a signal like S3, at 10, 15, 20 and $25 \mathrm{~Hz}$. The average response time is then below $1 \mathrm{~s}$, which seems again satisfactory in respect to the needs within the applications described in section II.

Eventually, it is interesting to notice that the response time of the subjects depends only slightly on the use of gloves The average response time to change from $\mathrm{S} 1$ to $\mathrm{S} 3$ is still below $1 \mathrm{~s}$, and $85 \%$ of response times of users to $\mathrm{S} 1$ are below $1 \mathrm{~s}$. Some testers reported the feeling of having improved their 
sensitivity with gloves due to skin pre-stretch, but there is no clear correlation with the response time.

As a summary, the use of 2 combined dynamic patterns, one of type S1 and the other one of type S3, appears of interest to provide tactile information to a user about two interesting types of information $\mathrm{S} 1$ and $\mathrm{S} 3$ and then respectively used for informing about tissue puncture and tissue nature. The switching between 4 frequencies $(10,15,20,25) \mathrm{Hz}$ should allow the user to get an information related to the 4 types of tissues, with acceptable response times. The subjective statements of the testers agree reasonable with the quantitive measurements of response time.

\section{CONClusion}

Robotized needle insertion is of great interest for development of percutaneous procedures as it can increase safety for the radiologist and the patient. With information on needletissue interaction at the robot level, it is possible to provide relevant information for needle insertion management which can be sensed by radiologist tactually. This paper focuses on how to provide two types of information, the occurence of puncture and the nature of tissues.

An experimental set-up was built in accordance with the scenario of a robotized puncturing procedure. Two signals are identified (S1 and S3) that offer reaction times close to $1 \mathrm{~s}$, defined as a requirement. Further tests are now needed to validate the results with a larger number of subjects, especially with skilled radiologists. The feedback strategy will then be implemented in the robotic system under development. This includes the automatic classification of organ according to the measured insertion forces during insertion. If response time becomes too high, needle speed might have to be reduced for safe insertion.

A longer-term perspective will deal with the integration of other information related to transverse forces, which could also be measured, to provide information for specific insertion situations like needle steering.

\section{ACKNOWLEDGMENT}

The authors would like to thank HelpTech $\mathrm{GmbH}$ for their support in building the experimental set-up.

\section{REFERENCES}

[1] P. R. Mueller and E. Van Sonnenberg, "Interventional Radiology in the Chest and Abdomen," New England Journal of Medicine, vol. 322, no. 19 , pp. 1364-1374, 1990.

[2] R. H. Kassamali and B. Ladak, "The role of robotics in interventional radiology: current status," Quantitative Imaging in Medicine and Surgery, vol. 5, pp. 340-343, June 2015.

[3] F. Cornelis, M. Havez, N. Lippa, S. Al-Ammari, D. Verdier, T. Carteret, N. Amoretti, A. Gangi, J. Palussiere, O. Hauger, and N. Grenier, "Radiologically guided percutaneous cryotherapy for soft tissue tumours: A promising treatment," Diagnostic and Interventional Imaging, vol. 94, no. 4, pp. 364 - 370, 2013.

[4] O. Piccin, P. Renaud, L. Barbé, B. Bayle, B. Maurin, and M. de Mathelin, "A Robotized Needle Insertion Device for Percutaneous Procedures," in Proceedings of ASME International Design Engineering Technical Conferences, vol. 7, pp. 443-440, 2005.

[5] A. Pfeil, L. Barbé, B. Wach, R. L. Cazzato, A. Gangi, and P. Renaud, "Observations and experiments for the definition of a new robotic device dedicated to ct, cbct and mri-guided percutaneous procedures," in International Conference of the IEEE Engineering in Medicine and Biology Society (EMBC), pp. 1708-1712, 2018.
[6] N. V. Tsekos, A. Khanicheh, E. Christoforou, and C. Mavroidis, "Magnetic resonance-compatible robotic and mechatronics systems for image-guided interventions and rehabilitation: a review study," Annual Review of Biomedical Engineering, vol. 9, pp. 351-387, 2007.

[7] W. Shang, H. Su, G. Li, and G. S. Fischer, "Teleoperation system with hybrid pneumatic-piezoelectric actuation for MRI-guided needle insertion with haptic feedback," IEEE International Conference on Intelligent Robots and Systems, pp. 4092-4098, 2013.

[8] I. Bricault, N. Zemiti, E. Jouniaux, C. Fouard, E. Taillant, F. Dorandeu, and P. Cinquin, "Light puncture robot for CT and MRI interventions," IEEE Engineering in Medicine and Biology Magazine, vol. 27, no. 3, pp. 42-50, 2008.

[9] N. Hungr, I. Bricault, P. Cinquin, and C. Fouard, "Design and Validation of a CT- and MRI-Guided Robot for Percutaneous Needle Procedures," IEEE Transactions on Robotics, vol. 32, no. 4, pp. 973987, 2016.

[10] S. Elayaperumal, J. H. Bae, B. L. Daniel, and M. R. Cutkosky, "Detection of membrane puncture with haptic feedback using a tipforce sensing needle," in International Conference on Intelligent Robots and Systems, pp. 3975-3981, 2014.

[11] "Worldwide cancer mortality statistics, Cancer Research UK," 2015.

[12] C. J. Walsh, N. C. Hanumara, A. H. Slocum, J.-A. Shepard, and R. Gupta, "A Patient-Mounted, Telerobotic Tool for CT-Guided Percutaneous Interventions," J. of Medical Devices, vol. 2, no. 1, p. 011007 , 2008.

[13] A. Pfeil, L. Barbé, B. Wach, A. Bruyas, F. Geiskopf, M. Nierenberger, and P. Renaud, "A 3d-printed needle driver based on auxetic structure and inchworm kinematics," in International Design Engineering Technical Conferences and Computers and Information in Engineering Conference, p. V05AT07A057, 2018.

[14] D. Rus and M. T. Tolley, "Design, fabrication and control of soft robots," Nature, vol. 521, pp. $467 \mathrm{EP}-, 052015$.

[15] C.-H. King, M. Culjat, M. Franco, J. Bisley, G. Carman, E. Dutson, and W. Grundfest, "A Multielement Tactile Feedback System for Robot-Assisted Minimally Invasive Surgery," IEEE Transactions on Haptics, vol. 2, no. 1, pp. 52-56, 2009

[16] S. Contu, C. M. L. Hughes, and L. Masia, "The Role of Visual and Haptic Feedback During Dynamically Coupled Bimanual Manipulation," IEEE Transactions on Haptics, vol. 9, no. 4, pp. 536-547, 2016.

[17] C. Gallacher, A. Mohtat, S. Ding, and J. Kövecses, "Toward opensource portable haptic displays with visual-force-tactile feedback colocation," IEEE Haptics Symposium, HAPTICS, vol. 2016-April, pp. 6571, 2016.

[18] J. H. Bae, A. K. Han, C. J. Ploch, B. L. Daniel, and M. R. Cutkosky, "Haptic feedback of membrane puncture with an MR-compatible instrumented needle and electroactive polymer display," 2017 IEEE World Haptics Conference, WHC 2017, pp. 54-59, 2017.

[19] I. Sarakoglou, N. Garcia-Hernandez, N. G. Tsagarakis, and D. G. Caldwell, "A high performance tactile feedback display and its integration in teleoperation," IEEE Transactions on Haptics, vol. 5, no. 3, pp. 252 263, 2012.

[20] J. Hergenhan, J. Rutschke, M. Uhl, S. E. Navarro, B. Hein, and H. Worn, "A haptic display for tactile and kinesthetic feedback in a CHAI 3D palpation training scenario," 2015 IEEE International Conference on Robotics and Biomimetics, IEEE-ROBIO 2015, pp. 291296, 2015.

[21] M. Li, S. Luo, T. Nanayakkara, L. D. Seneviratne, P. Dasgupta, and K. Althoefer, "Multi-fingered haptic palpation using pneumatic feedback actuators," Sensors and Actuators, A: Physical, vol. 218, pp. 132-141, 2014.

[22] Z. F. Quek, S. B. Schorr, I. Nisky, W. R. Provancher, and A. M. Okamura, "Sensory Substitution and Augmentation of Forces and Torques Using Tactile Skin Deformation Feedback," Transactions on Haptics, vol. 8, no. 2, pp. 209-221, 2015.

[23] D. E. Fry, W. E. Harris, E. N. Kohnke, and C. L. Twomey, "Influence of double-gloving on manual dexterity and tactile sensation of surgeons," Journal of the American College of Surgeons, vol. 210, no. 3, pp. 325$330,2010$.

[24] C. Hatzfeld and T. A. Kern, eds., Engineering Haptic Devices. Springer, 2014.

[25] H. P. E. in Chief), S. Y. (Editor), D. M. (Editor), R. G. (Editor), and J. T. W. (Editor), eds., Stevens' handbook of experimental psychology. John Wiley \& Sons, Inc, 3rd edition ed., 2004. 